\title{
How Infection with C. difficile Infection Aggravates Inflammatory Bowel Disease: Is It CDI or the CDAl?
}

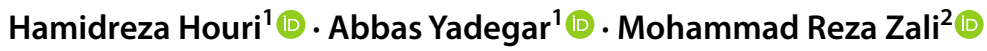

Accepted: 6 October 2020 / Published online: 23 October 2020

(c) Springer Science+Business Media, LLC, part of Springer Nature 2020

Inflammatory bowel disease (IBD) is regarded as a lifelong, relapsing-remitting inflammatory disorder of the gastrointestinal (GI) tract, with an unknown etiology and unpredictable clinical course [1]. GI infections commonly trigger relapse in patients with IBD; among these infections, Clostridioides (formerly Clostridium) difficile infection (CDI) is reported as the most common GI infection in patients with IBD, with recurrence in up to one-third of patients. CDI frequently complicates the subsequent course of IBD, delays IBD remission, and increases the need for colectomy, and even increases mortality rates [2]. Risk factors contributing to the acquisition and severity of CDI in IBD are preexisting colonic inflammation, especially in ulcerative colitis (UC), concomitant immunosuppression, prolonged hospitalization, and long-term antibiotic use. Though the clinical manifestations of an IBD flare and CDI have considerable overlap, the treatment strategies are markedly different with escalation of immunosuppression for an IBD flare compared with reduction in immunosuppression and antibiotics for active CDI [3]. Although IBDassociated inflammation could predispose patients to CDI, CDI may exacerbate IBD by triggering a mucosal inflammatory response or by reducing the diversity of the luminal microbiota [4]. Accordingly, whether IBD disease severity predisposes to the development of the CDI or whether CDI exacerbates IBD has often been presented as a problem of origin and first cause.

Abbas Yadegar

a.yadegar@sbmu.ac.ir; babak_y1983@yahoo.com

1 Foodborne and Waterborne Diseases Research Center, Research Institute for Gastroenterology and Liver Diseases, Shahid Beheshti University of Medical Sciences, Tehran, Iran

2 Gastroenterology and Liver Diseases Research Center, Research Institute for Gastroenterology and Liver Diseases, Shahid Beheshti University of Medical Sciences, Tehran, Iran
In this issue of Digestive Diseases and Sciences, Varma and colleagues [5] retrospectively evaluated patients hospitalized for $\geq 2$ IBD flares at New York-Presbyterian Columbia University Irving Medical Center from January 2010 to September 2019. The authors compared the time to IBD flare between patients who were hospitalized for a flare complicated by CDI and subsequently for a CDInegative flare $( \pm$; cohort $A)$ versus patients who were hospitalized for two CDI-negative flares (-I-; cohort B). They also determined the time between flares among the subset of cohort A patients who had three flares $(-/ \pm$; cohort $C)$ before and after CDI. As a result, they reported that patients with prior CDI had significantly shorter time to IBD relapse compared with those without any history of CDI. On the contrary, among patients with IBD who had CDI, the interval between flares was similar before CDI compared with afterward. Their findings suggest that the acquisition of CDI may not inevitably influence the course of IBD, but rather, the shorter time to disease relapse among patients with CDI may be related to the intrinsic disease and/or patient characteristics that predispose patients to CDI, and ultimately impact the time to flare. Also, the authors found that patients with IBD who acquired CDI had a significantly lower BMI and higher Charlson comorbidity index at baseline, consistent with augmented illness severity and GI inflammation in these patients. In contrary, CDI-negative patients were more likely to have received budesonide and less likely to have received biologics prior to their first IBD-related hospitalization. Moreover, CDI-negative patients were more likely to have an escalation in IBD therapy after hospitalization. Therefore, these findings corroborate the hypothesis that patients with severe IBD are more likely to develop CDI.

Some previous studies have indicated that IBD itself is a risk factor for acquiring CDI, particularly among patients with colonic involvement [6]. Moreover, patients with IBD regularly receive broad-spectrum antibiotics as a part of the treatment of disease exacerbations or have complications from immunosuppressive therapy. Hence, these patients are 
regarded as a particular high-risk population for the development of CDI [6]. Accordingly, routine empirical therapy with broad-spectrum antibiotics in patients with IBD should be discouraged. Furthermore, biologic therapy in IBD, specifically TNF $\alpha$ inhibitors, increases the risk of CDI among IBD patients [6, 7].

In the present study, Varma et al. [5] indicated that patients with prior CDI had a significantly shorter time to disease relapse and inferior outcomes compared with their CDI-negative counterparts, in which the presence of CDI was significantly associated with hospitalization at the time of the second IBD flare. These observations are in accordance with prior studies that have demonstrated the risk of poorer outcomes in IBD-CDI when compared with either condition alone [8]. Nevertheless, although CDI was reported to be associated with a shorter time to flare in this study, the authors assumed that it is more likely due to more severe IBD rather than acquisition of CDI, since all IBDCDI patients were negative for $C$. difficile at the time of the second flare. Moreover, the exploratory analysis of patients who had three hospitalizations for flares in whom CDI testing was negative, then became positive, then turned negative during the flare hospitalizations, showed no difference in the time to flare before or after acquiring CDI.

The results of the current study suggest that CDI may not necessarily worsen the outcomes of IBD, but rather that severe IBD predisposes to CDI and therefore is associated with inferior clinical outcomes, including earlier time to IBD relapse. Nonetheless, several questions deserve attention. The authors do not provide evidence sufficient to contradict previous reports that supported significant adverse impact of CDI on patients with IBD. Patients with both CDI and IBD have a sixfold increase in emergency GI surgery or colectomy and have a greater mortality rate compared with those with IBD alone [4]. Jodorkovsky et al. [9] previously reported a higher rate of UC-related emergency hospitalizations one year after an initial episode of CDI with a twofold increase in the rate of colectomy. Jen and colleagues [10], using data from the National Health Service in England, found a nearly sixfold excess mortality after the initial hospitalization for CDI.

In summary, while the authors found some evidence to suggest that acquiring CDI may not necessarily affect the course of IBD, patients with IBD co-infected with $C$. difficile require escalation of medical therapy and have a higher adjusted risk of mortality compared with patients with IBD alone. Due to the controversial data provided by retrospective studies, further prospective cohorts would better evaluate the impact of CDI on IBD outcomes. Finally, clinical trials involving fecal microbiota transplantation (FMT) in patients with IBD with CDI could also better assess the impact of CDI and its treatment on IBD disease course.

\section{References}

1. Wilson JC, Furlano RI, Jick SS, Meier CR. Inflammatory bowel disease and the risk of autoimmune diseases. J Crohn's Colitis. 2016;10:186-193.

2. Rodemann JF, Dubberke ER, Reske KA, Seo DH, Stone CD. Incidence of Clostridium difficile infection in inflammatory bowel disease. Clin Gastroenterol Hepatol Off Clin Pract J Am Gastroenterol Assoc.. 2007;5:339-344.

3. Issa M, Ananthakrishnan AN, Binion DG. Clostridium difficile and inflammatory bowel disease. Inflamm Bowel Dis. 2008;14:1432-1442.

4. Trifan A, Stanciu C, Stoica O, Girleanu I, Cojocariu C. Impact of Clostridium difficile infection on inflammatory bowel disease outcome: a review. World J Gastroenterol. 2014;20:11736-11742.

5. Varma S, Faye AS, Kannan A, et al. Patients with more severe IBD get clostridioides difficile rather than clostridioides difficile increasing the severity of IBD. Dig Dis Sci. (Epub ahead of print). https://doi.org/10.1007/s10620-020-06504-y.

6. Balram B, Battat R, Al-Khoury A, et al. Risk factors associated with Clostridium difficile infection in inflammatory bowel disease: a systematic review and meta-analysis. J Crohn's Colitis. 2019;13:27-38.

7. Gholam-Mostafaei FS, Yadegar A, Aghdaei HA, Azimirad M, Daryani NE, Zali MR. Anti-TNF containing regimens may be associated with increased risk of Clostridioides difficile infection in patients with underlying inflammatory bowel disease. Curr Res Transl Med. 2020;68:125-130.

8. Nitzan O, Elias M, Chazan B, Raz R, Saliba W. Clostridium difficile and inflammatory bowel disease: role in pathogenesis and implications in treatment. World $J$ Gastroenterol. 2013;19:7577-7585.

9. Jodorkovsky D, Young Y, Abreu MT. Clinical outcomes of patients with ulcerative colitis and co-existing Clostridium difficile infection. Dig Dis Sci. 2010;55:415-420. https://doi.org/10.1007/ s10620-009-0749-9.

10. Jen MH, Saxena S, Bottle A, Aylin P, Pollok RC. Increased health burden associated with Clostridium difficile diarrhoea in patients with inflammatory bowel disease. Aliment Pharmacol Ther. 2011;33:1322-1331.

Publisher's Note Springer Nature remains neutral with regard to jurisdictional claims in published maps and institutional affiliations. 